\title{
Fuglede-type decompositions of representations
}

\author{
by \\ MAREK Kosiek (Kraków)
}

\begin{abstract}
It is shown that reducing bands of measures yield decompositions not only of an operator representation itself, but also of its commutant. This has many consequences for commuting Hilbert space representations and for commuting operators on Hilbert spaces. Among other things, it enables one to construct a Lebesgue-type decomposition of several commuting contractions without assuming any von Neumann-type inequality.
\end{abstract}

1. Introduction and preliminaries. The method of decompositions of function algebra representations without using any dilations was developed by Mlak in the seventies. Among other things, it made possible constructions of representations analogous to the Nagy-Foias functional calculus for completely nonunitary contractions. The decompositions with respect to nontrivial Gleason parts were later extended by Szafraniec to the more general setting (equivalent to reducing bands of measures). The ideas of Mlak and Szafraniec have had numerous applications in the theory of operator representations. In this paper we show how their ideas can be applied to obtain a decomposition of the commutant useful in some new applications.

The paper is organized as follows: The present section contains preliminaries on bands of measures and operator representations of function algebras. The beginning of Section 2 recalls band decompositions of operator representations due to Mlak [11] and Szafraniec [16]. The rest of it is devoted to band decompositions of the commutant. In Section 3 we consider band decompositions of mutually commuting representations. The results obtained are illustrated by an application.

A Lebesgue-type decomposition of $N$-tuples of commuting contractions presented in Section 4 is another application of the results proved in Section 3. We get it without requiring any kind of von Neumann inequality. Finally, in Section 5 some absolute continuity and singularity properties of

2000 Mathematics Subject Classification: Primary 47A60; Secondary 47A15, 46J10, $46 \mathrm{~J} 25$.

Key words and phrases: band of measures, representations, commutant, contractions, hyperinvariant subspaces. 
commuting contractions are considered. We also obtain a result concerning hyperinvariant subspaces which is connected with the above mentioned properties.

Let $X$ be a compact Hausdorff space. We denote by $C(X)$ the Banach algebra of all complex continuous functions on $X$ equipped with the supnorm, and by $M(X)$ the set of all complex Borel measures on $X$. Recall that $M(X)$ is a Banach space with the total variation norm. If $E$ is a subset of $M(X)$ then $E^{\mathrm{s}}$ will denote the set of all measures on $X$ singular to each measure in $E$. A subset $\mathcal{M}$ of $M(X)$ is a band (see [7, Sec. 2]) if $\left(\mathcal{M}^{\mathrm{s}}\right)^{\mathrm{s}}=\mathcal{M}$. It is easy to see that any band is a closed subspace of $M(X)$ which is also closed with respect to the absolute continuity. Any measure $\mu \in M(X)$ has a Lebesgue decomposition of the form

$$
\mu=\mu^{\mathcal{N}}+\mu^{\mathcal{M} \mathfrak{M}^{\mathrm{s}}}
$$

where $\mu^{\mathcal{M}} \in \mathcal{M}$ and $\mu^{\mathcal{M}^{\mathrm{s}}} \in \mathcal{M}^{\mathrm{s}}$. (For details and terminology concerning bands we refer the reader to [3, Sec. 20], [4, V.17]). In the case when only one band is considered we will write $\mu^{\mathrm{s}}$ instead of $\mu^{\mathcal{M N}^{\mathrm{s}}}$ for the singular part of $\mu$.

In what follows, assume that $A$ is an arbitrary function algebra on $X$, i.e. a uniformly closed subalgebra of $C(X)$ containing constants and separating the points of $X$. We say that a measure $\mu$ is orthogonal to $A$ (or annihilates $A$ ) if $\int u d \mu=0$ for $u \in A$. The set of all such measures is denoted by $A^{\perp}$.

Let $\mathcal{H}$ be a complex Hilbert space with inner product $(\cdot, \cdot)$ and norm $\|\cdot\|$. We denote by $L(\mathcal{H})$ the algebra of all bounded linear transformations acting on $\mathcal{H}$, and by $I$ the identity operator on $\mathcal{H}$. A linear mapping $T: A \rightarrow L(\mathcal{H})$ is called a representation of a function algebra $A$ if

$$
T(u v)=T(u) T(v) \quad \text { for } u, v \in A
$$

and there is a positive constant $k$ such that

$$
\|T(u)\| \leq k\|u\|, \quad u \in A .
$$

Without loss of generality we may always assume that $T(1)=I$.

It is a consequence of standard techniques (see [11]) that for each $x, y \in \mathcal{H}$ there exists a complex, Borel, regular measure $\mu_{x, y}$ on $X$ such that

$$
(T(u) x, y)=\int u d \mu_{x, y} \quad \text { for } x, y \in \mathcal{H}, u \in A
$$

and

$$
\left\|\mu_{x, y}\right\| \leq k\|x\| \cdot\|y\| .
$$

The measures $\mu_{x, y}$ are called elementary measures of $T$. 
2. Decomposition of the commutant. A band $\mathcal{M} \subset M(X)$ is said to be reducing with respect to $A$ if for every measure $\mu \in A^{\perp}$ we have $\mu_{\mathcal{M}} \in A^{\perp}$. (When $A$ is fixed we say simply that $\mathcal{M}$ is reducing.) Suppose we are given a representation $T: A \rightarrow L(\mathcal{H})$ satisfying (1.3). Let $\mathcal{M}$ be a band in $M(X)$. Let $\left\{\mu_{x, y}\right\}_{x, y \in \mathcal{H}}$ be a collection of its elementary measures. Each of the measures $\mu_{x, y}$ has a Lebesgue decomposition (1.1) with respect to $\mathcal{N}$. By (1.4) we have

$$
(T(u) x, y)=\int u d \mu_{x, y}^{\mathcal{X}}+\int u d \mu_{x, y}^{\mathrm{s}},
$$

where $\mu_{x, y}^{\mathcal{X}} \in \mathcal{M}$ and $\mu_{x, y}^{\mathrm{s}} \in \mathcal{M}^{\mathrm{s}}$. It is shown in [11] and [4] that with any $u \in A$ we can associate bounded linear operators $T^{\mathcal{M}}(u), T^{\mathrm{s}}(u)$ (the last one denoted by $T^{\mathcal{M}^{\mathrm{s}}}(u)$ in the general situation) such that

$$
\left(T^{\mathcal{M}}(u) x, y\right)=\int u d \mu_{x, y}^{\mathcal{N}}, \quad\left(T^{\mathrm{s}}(u) x, y\right)=\int u d \mu_{x, y}^{\mathrm{s}} \quad \text { for } x, y \in \mathcal{H} .
$$

Moreover (see [11, Thm. 2.1], [16, Thm. 3.2]), we have the following

Proposition 2.1. Let $\mathcal{H}_{\mathcal{M}}=T^{\mathcal{M}}(1) \mathcal{H}, \mathcal{H}_{\mathrm{s}}=T^{\mathrm{s}}(1) \mathcal{H}$. The mappings

$$
T^{\mathcal{M}}: A \ni u \mapsto T^{\mathcal{M}}(u) \in L\left(\mathcal{H}_{\mathcal{M}}\right), \quad T^{\mathrm{s}}: A \ni u \mapsto T^{\mathrm{s}}(u) \in L\left(\mathcal{H}_{\mathrm{s}}\right)
$$

are representations with $\left\|T^{\mathcal{M}}(u)\right\| \leq k\|u\|,\left\|T^{\mathrm{s}}(u)\right\| \leq k\|u\|$ for $u \in A$. The space $\mathcal{H}$ splits into the direct sum of subspaces invariant for $T$ :

$$
\mathcal{H}=\mathcal{H}_{\mathcal{M}}+\mathcal{H}_{\mathrm{s}}
$$

and $T^{\mathcal{M}}(u)=\left.T(u)\right|_{\mathcal{H}_{\mathfrak{M}}}, T^{\mathrm{s}}(u)=\left.T(u)\right|_{\mathcal{H}_{\mathrm{s}}}$ for $u \in A$. In the case of $k=1$ the sum (2.7) is orthogonal, $T^{\mathcal{M}}(1), T^{\mathrm{s}}(1)$ are orthogonal projections, and the subspaces of the decomposition (2.7) reduce $T$.

We call $T^{\mathcal{M}}$ the absolutely continuous, and $T^{\mathrm{s}}$ the singular part of $T$ with respect to $\mathcal{M}$. The notions of $T^{\mathcal{M}}(u), T^{\mathrm{s}}(u)$ have double meanings. In (2.6) they act on $\mathcal{H}$ but in Proposition 2.1 they are considered as operators on the subspaces $\mathcal{H}_{\mathcal{M}}, \mathcal{H}_{\mathrm{s}}$ of $\mathcal{H}$. There is no misunderstanding here. Since $T^{\mathcal{N}}$, $T^{\mathrm{s}}$ are multiplicative, the operators $T^{\mathcal{N}}(1), T^{\mathrm{s}}(1)$ are projections. Hence for each $u \in A$ the subspaces $\mathcal{H}_{\mathcal{M}}, \mathcal{H}_{\mathrm{s}}$ are invariant for $T^{\mathcal{M}}(u), T^{\mathrm{s}}(u)$, and $T^{\mathcal{M}}(u)$ is a zero operator on $\mathcal{H}_{\mathrm{s}}$, while $T^{\mathrm{s}}(u)$ is a zero operator on $\mathcal{H}_{\mathcal{M}}$.

Now we show that the decomposition given by Proposition 2.1 decomposes also the commutant of $T$. We say that $W \in L(\mathcal{H})$ commutes with the representation $T$ if $W T(u)=T(u) W$ for all $u \in A$.

Theorem 2.2. Assume $A$ is a function algebra on $X, \mathcal{M} \subset M(X)$ is a reducing band with respect to $A$, and $T: A \rightarrow L(\mathcal{H})$ is a representation satisfying (1.3). If $W \in L(\mathcal{H})$ commutes with $T$, then $W$ also commutes with its absolutely continuous part $T^{\mathcal{N}}$ and singular part $T^{\mathcal{M}^{\mathrm{s}}}$.

The subspaces $\mathcal{H}_{\mathcal{M}}$ and $\mathcal{H}_{\mathrm{s}}$ in the decomposition (2.7) are invariant for $W$. Moreover, they are mutually orthogonal and reducing for $W$ when $k=1$ in (1.3). 
Proof. Let $u \in A$ and $x, y \in \mathcal{H}$. Then

$$
\begin{aligned}
(W T(u) x, y) & =\left(T^{\mathcal{M}}(u) x, W^{*} y\right)+\left(T^{\mathrm{s}}(u) x, W^{*} y\right) \\
& =\int u d \mu_{x, W^{*} y}^{\mathcal{N}}+\int u d \mu_{x, W^{*} y}^{\mathrm{s}}, \\
(W T(u) x, y) & =(T(u) W x, y)=\left(T^{\mathcal{N}}(u) W x, y\right)+\left(T^{\mathrm{s}}(u) W x, y\right) \\
& =\int u d \mu_{W x, y}^{\mathcal{N}}+\int u d \mu_{W x, y}^{\mathrm{s}} .
\end{aligned}
$$

Hence the measure

$$
\mu_{x, W^{*} y}^{\mathcal{X}}-\mu_{W x, y}^{\mathcal{N}}+\mu_{x, W^{*} y}^{\mathrm{S}}-\mu_{W x, y}^{\mathrm{s}}
$$

is orthogonal to $A$, and consequently so is its absolutely continuous part $\mu_{x, W^{*} y}^{\mathcal{N}}-\mu_{W x, y}^{\mathcal{M}}$ and singular part $\mu_{x, W^{*} y}^{\mathrm{s}}-\mu_{W x, y}^{\mathrm{s}}$, since $\mathcal{M}$ is reducing. So

$$
\begin{aligned}
\left(W T^{\mathcal{M}}(u) x, y\right) & =\left(T^{\mathcal{N}}(u) x, W^{*} y\right)=\int u d \mu_{x, W^{*} y}^{\mathcal{X}} \\
& =\int u d \mu_{W x, y}^{\mathcal{M}}=\left(T^{\mathcal{M}}(u) W x, y\right), \\
\left(W T^{\mathrm{s}}(u) x, y\right) & =\left(T^{\mathrm{s}}(u) x, W^{*} y\right)=\int u d \mu_{x, W^{*} y}^{\mathrm{s}} \\
& =\int u d \mu_{W x, y}^{\mathrm{s}}=\left(T^{\mathrm{s}}(u) W x, y\right) .
\end{aligned}
$$

Since $x, y$ are arbitrary, we get

$$
W T^{\mathcal{M}}(u)=T^{\mathcal{M}}(u) W, \quad W T^{\mathrm{s}}(u)=T^{\mathrm{s}}(u) W \quad \text { for } u \in A,
$$

which completes the proof.

Consider now the case when we have a second function algebra $B$ on a compact set $Y$ and its operator representation $W: B \rightarrow L(\mathcal{H})$ satisfying the condition

$$
\|W(v)\| \leq l\|v\|, \quad v \in B
$$

and commuting with $T$, i.e.

$$
T(u) W(v)=W(v) T(u), \quad u \in A, v \in B .
$$

By Theorem 2.2 the representation $W$ also commutes with $T^{\mathcal{M}}$. Now define

$$
W^{\mathcal{M}}(v):=T^{\mathcal{M}}(1) W(v), \quad W^{\mathrm{s}}(v):=T^{\mathrm{s}}(1) W(v) \quad \text { for } v \in B .
$$

It can be easily seen that $W^{\mathcal{M}}: B \rightarrow L\left(\mathcal{H}_{\mathcal{M}}\right)$ is a linear mapping and

$$
\left\|W^{\mathcal{M}}(v)\right\| \leq\left\|T^{\mathcal{M}}(1)\right\| \cdot\|W(v)\| \leq k l\|v\| \quad \text { for } v \in B .
$$

Let now $u, v \in B$. Then 


$$
\begin{aligned}
W^{\mathcal{M}}(u v) & =T^{\mathcal{M}}(1) W(u v)=T^{\mathcal{M}}(1) W(u) W(v) \\
& =T^{\mathcal{M}}(1) T^{\mathcal{M}}(1) W(u) W(v) \\
& =T^{\mathcal{M}}(1) W(u) T^{\mathcal{M}}(1) W(v)=W^{\mathcal{M}}(u) W^{\mathcal{M}}(v) .
\end{aligned}
$$

Similar considerations apply to $W^{\mathrm{s}}$. It is easy to see that $W^{\mathcal{M}}(1)=T^{\mathcal{M}}(1)$ and $W^{\mathrm{s}}(1)=T^{\mathrm{s}}(1)$.

So we have the following:

Theorem 2.3. Let $A, T$ and $\mathcal{M}$ be as in Theorem 2.2. Let $W$ be a representation of a function algebra $B$ satisfying (2.8) and commuting with $T$. Then there exist representations

$$
W^{\mathcal{N}}: B \rightarrow L\left(\mathcal{H}_{\mathcal{M}}\right), \quad W^{\mathrm{s}}: B \rightarrow L\left(\mathcal{H}_{\mathrm{s}}\right)
$$

commuting with $T^{\mathcal{M}}$ and $T^{\mathrm{s}}$, satisfying

$$
\left\|W^{\mathcal{M}}(v)\right\| \leq k l\|v\|, \quad\left\|W^{\mathrm{s}}(v)\right\| \leq k l\|v\| \quad \text { for } v \in B
$$

and such that

$$
W=W^{\mathcal{M}}+W^{\mathrm{s}} \text {. }
$$

If moreover $k=1$, then the decomposition (2.11) is orthogonal.

3. Commuting representations. Let us now consider the case of two commuting representations $T_{i}: A_{i} \rightarrow L(\mathcal{H}), i=1,2$, with $\left\|T_{i}(u)\right\| \leq k_{i}\|u\|$, where $A_{i}$ is a function algebra on a compact set $X_{i}$ with a reducing band $\mathcal{M}_{i} \subset M\left(X_{i}\right)$. For $T_{1}, T_{2}$ take now the decompositions with respect to the bands $\mathcal{M}_{1}, \mathcal{M}_{2}$ respectively, given by Proposition 2.1 , and put

$$
\begin{array}{rlrl}
P_{\mathrm{aa}} & :=T_{1}^{\mathcal{M}_{1}}(1) T_{2}^{\mathcal{\mathcal { N } _ { 2 }}}(1), & P_{\mathrm{as}}:=T_{1}^{\mathcal{N}_{1}}(1) T_{2}^{\mathrm{s}}(1), \\
P_{\mathrm{sa}}:=T_{1}^{\mathrm{s}}(1) T_{2}^{\mathcal{\mathcal { M } _ { 2 }}}(1), & P_{\mathrm{ss}}:=T_{1}^{\mathrm{s}}(1) T_{2}^{\mathrm{s}}(1) .
\end{array}
$$

By Theorem 2.2, for $u \in A_{1}, v \in A_{2}$ all the operators $T_{1}^{\mathcal{N}_{1}}(u), T_{1}^{\mathrm{s}}(u)$, $T_{2}^{\mathcal{N}_{2}}(v), T_{2}^{\mathrm{s}}(v)$ commute (considered as operators on the whole space). Hence the norm of each operator defined in (3.12) is less than or equal to $k_{1} k_{2}$ and all of them are projections. Define now

$$
\begin{array}{lll}
T_{1}^{\mathrm{aa}}(u):=P_{\mathrm{aa}} T_{1}(u), & T_{1}^{\mathrm{as}}(u):=P_{\mathrm{as}} T_{1}(u), & \\
T_{1}^{\mathrm{sa}}(u):=P_{\mathrm{sa}} T_{1}(u), & T_{1}^{\mathrm{ss}}(u):=P_{\mathrm{ss}} T_{1}(u) & \text { for } u \in A_{1}, \\
T_{2}^{\mathrm{aa}}(v):=P_{\mathrm{aa}} T_{2}(v), & T_{2}^{\mathrm{as}}(v):=P_{\mathrm{as}} T_{2}(v), & \\
T_{2}^{\mathrm{sa}}(v):=P_{\mathrm{sa}} T_{2}(v), & T_{2}^{\mathrm{ss}}(v):=P_{\mathrm{ss}} T_{2}(v) & \text { for } v \in A_{2} .
\end{array}
$$

As in (2.10), we see that the mapping $T_{1}^{\mathrm{aa}}: A_{1} \rightarrow \mathcal{H}_{\mathrm{aa}}:=P_{\mathrm{aa}}(\mathcal{H})$ is a representation and by the definition of $P_{\text {aa }}$ we have $T_{1}^{\mathrm{aa}}(u)=T_{2}^{\mathcal{\mathcal { N } _ { 2 }}}(1) T_{1}^{\mathcal{M}_{1}}(u)$ for $u \in A_{1}$, and hence $\left\|T_{1}^{\mathrm{aa}}(u)\right\| \leq k_{1} k_{2}\|u\|$. The same holds for all other mappings defined in (3.13). 
Then we have the following

TheOREM 3.1. For $i=1,2$ let $T_{i}: A_{i} \rightarrow L(\mathcal{H})$ be a representation of a function algebra $A_{i}$ on a compact set $X_{i}$ with reducing bands $\mathcal{M}_{i} \subset M\left(X_{i}\right)$. Assume that $T_{1}, T_{2}$ commute, i.e. $T_{1}(u) T_{2}(v)=T_{2}(v) T_{1}(u)$ for $u \in A_{1}$, $v \in A_{2}$, and satisfy the estimates $\left\|T_{i}(u)\right\| \leq k_{i}\|u\|$ for $u \in A_{i}, i=1,2$. Then for $i=1,2$ we have the decomposition

$$
T_{i}=T_{i}^{\mathrm{aa}}+T_{i}^{\mathrm{as}}+T_{i}^{\mathrm{sa}}+T_{i}^{\mathrm{ss}}
$$

corresponding to the following decomposition of the space $\mathcal{H}$ into closed subspaces invariant for $T_{i}$ :

$$
\mathcal{H}=\mathcal{H}_{\mathrm{aa}}+\mathcal{H}_{\mathrm{as}}+\mathcal{H}_{\mathrm{sa}}+\mathcal{H}_{\mathrm{ss}},
$$

where $\mathcal{H} . .=P . . \mathcal{H}$. Every part of the decomposition (3.14) is a representation of $A_{i}$ with values in the appropriate part of (3.15). Moreover,

$$
\left\|T_{i}^{*}(u)\right\| \leq k_{1} k_{2}\|u\|, \quad u \in A_{i} .
$$

In the case when $k_{1}=k_{2}=1$, the decompositions (3.14) and (3.15) are orthogonal.

We can obtain a more specific decomposition of the type (3.15) if $A_{1}$ is the ball algebra $A\left(\mathbb{B}_{n}\right)$, where $\mathbb{B}_{n}=\left\{\left(z_{1}, \ldots, z_{n}\right) \in \mathbb{C}^{n}: \sum_{i=1}^{n}\left|z_{i}\right|^{2}<1\right\}$, and $A_{2}$ is the bidisc algebra $A\left(\mathbb{D}^{2}\right)$. Examples of representations of $A\left(\mathbb{B}_{n}\right)$ are provided by spherical contractions. These are the commutative $n$-tuples $\left(S_{1}, \ldots, S_{n}\right)$ in $L(\mathcal{H})$ such that $\left\|S_{1} x\right\|^{2}+\ldots+\left\|S_{n} x\right\|^{2} \leq\|x\|^{2}$ for $x \in \mathcal{H}$, or equivalently $\Delta_{S}^{(1)}:=I-S_{1}^{*} S_{1}-\ldots-S_{n}^{*} S_{n} \geq 0$. Assuming the positivity of a similarly defined $\Delta_{S}^{(n)}$, Müller and Vasilescu ([13, Thm. 11]) show that $S_{1}, \ldots, S_{n}$ dilate to commuting normal operators $\widetilde{S}_{1}, \ldots, \widetilde{S}_{n}$ on a Hilbert space $\mathcal{K} \supset \mathcal{H}$ such that the joint left spectrum $\sigma_{l}\left(\widetilde{S}_{1}, \ldots, \widetilde{S}_{n}\right)$ lies in the unit sphere $\partial \mathbb{B}_{n}$. It is well known that all types of joint spectra coincide on normal tuples (cf. Uniqueness Theorem in [15]). Therefore the support of the joint spectral measure of $\widetilde{S}_{1}, \ldots, \widetilde{S}_{n}$ lies in $\partial \mathbb{B}_{n}$ and we can extend the natural action of the polynomials: $\mathbb{C}\left[z_{1}, \ldots, z_{n}\right] \ni p \mapsto p\left(S_{1}, \ldots, S_{n}\right)$ to a contractive representation

$$
T_{1}: A\left(\mathbb{B}_{n}\right) \rightarrow L(\mathcal{H}), \quad T_{1}\left(z_{j}\right)=S_{j}, \quad j=1, \ldots, n .
$$

Assume now that $T_{2}$ is a contractive bidisc algebra representation $T_{2}: A\left(\mathbb{D}^{2}\right) \rightarrow L(\mathcal{H})$ given by an arbitrary pair of commuting contractions $R_{1}=T_{2}\left(z_{1}\right), R_{2}=T_{2}\left(z_{2}\right)$. (Its existence follows from the Ando Dilation Theorem [1].)

In this context we are interested in the decomposition with respect to the bands of annihilating measures $\mathcal{M}_{1}=A\left(\mathbb{B}_{n}\right)^{\perp}, \mathcal{M}_{2}=A\left(\mathbb{D}^{2}\right)^{\perp}$. Note that, by the Henkin theorem (see [14, Thm. 9.3.1]) together with the Cole-Range theorem (see [14, Thm. 9.6.1]), $\mathcal{M}_{1}$ is equal to the band generated by all 
the measures representing points in $\mathbb{B}_{n}$. By $[5, \mathrm{VI} .1 .2]$, each measure in $\mathcal{M}_{1}$ is absolutely continuous with respect to some measure representing the evaluation at the origin for the algebra $A\left(\mathbb{B}_{n}\right)$.

On the other hand, the band $\mathcal{M}_{2}$ decomposes into a direct sum of three mutually singular reducing bands described in [2, Thm. 1]. A successive application of the above properties and of the preceding theorem gives

Corollary 3.2. Assume $T_{1}: A\left(\mathbb{B}_{n}\right) \rightarrow L(\mathcal{H})$ and $T_{2}: A\left(\mathbb{D}^{2}\right) \rightarrow L(\mathcal{H})$ are commuting representations obtained in the way just described. Then $\mathcal{H}$ decomposes orthogonally into eight direct summands

$$
\mathcal{H}=\mathcal{H}_{\mathrm{a} 0} \oplus \mathcal{H}_{\mathrm{a} 1} \oplus \mathcal{H}_{\mathrm{a} 2} \oplus \mathcal{H}_{\mathrm{a} 3} \oplus \mathcal{H}_{\mathrm{s} 0} \oplus \mathcal{H}_{\mathrm{s} 1} \oplus \mathcal{H}_{\mathrm{s} 2} \oplus \mathcal{H}_{\mathrm{s} 3}
$$

so that each $\mathcal{H}$., reduces $T_{1}, T_{2}$, and:

(1) There exists a collection of elementary measures of $\left.T_{1}\right|_{\mathcal{H}_{\mathrm{a}}}$. each of which is absolutely continuous with respect to some measure representing the evaluation at the origin for the algebra $A\left(\mathbb{B}_{n}\right)$.

(2) There exists a unique spectral measure of $\left.T_{1}\right|_{\mathcal{H}_{s}}$ on $\partial \mathbb{B}_{n}$, and it is singular to $A\left(\mathbb{B}_{n}\right)^{\perp}$.

(3) There exists a collection of elementary measures of $\left.T_{2}\right|_{\mathcal{H}_{0} \text { o }}$ each of which is absolutely continuous with respect to some measure representing the evaluation at the origin for the algebra $A\left(\mathbb{D}^{2}\right)$.

(4) There exists a collection of elementary measures of $\left.T_{2}\right|_{\mathcal{H}_{\cdot 1}}$ (resp. $\left.\left.T_{2}\right|_{\mathcal{H}_{.2}}\right)$ each of which is supported on a set of the form $E \times \partial \mathbb{D}($ resp. $\partial \mathbb{D} \times$ $E)$, where $E$ is a nullset for $A(\mathbb{D})^{\perp}$, and its projection onto the second (resp. first) component is absolutely continuous with respect to the Lebesgue measure on $\partial \mathbb{D}$.

(5) There exists a unique spectral measure of $\left.T_{2}\right|_{\mathcal{H}_{\cdot 3}}$ on $\partial \mathbb{D}^{2}$, and it is singular to $A\left(\mathbb{D}^{2}\right)^{\perp}$.

Consider now the case of $N$ commuting representations $T_{i}: A_{i} \rightarrow L(\mathcal{H})$, $i=1, \ldots, N$, with $\left\|T_{i}(u)\right\| \leq k_{i}\|x\|$, where $A_{i}$ is a function algebra on a compact set $X_{i}$ with a reducing band $\mathcal{M}_{i} \subset M\left(X_{i}\right)$. Using similar ideas to (3.12), we can define a finite family of projections $\left\{P_{\alpha}\right\}$ on $\mathcal{H}$ such that for every $\alpha$ we have $P_{\alpha}=S_{1}(1) \ldots S_{N}(1)$, where $S_{i}$ is $T_{i}^{\mathcal{M}_{i}}$ or $T_{i}^{\mathcal{N}_{i}^{\mathrm{s}}}$. Now defining the parts of each representation $T_{i}$ as in (3.13) we get

ThEOREM 3.3. Let $T_{i}: A_{i} \rightarrow L(\mathcal{H}), i=1, \ldots, N$, be a representation of a function algebra $A_{i}$ on a compact set $X_{i}$ with a reducing band $\mathcal{M}_{i} \subset$ $M\left(X_{i}\right)$. Assume that $T_{1}, \ldots, T_{N}$ commute, i.e. $T_{i}(u) T_{j}(v)=T_{j}(v) T_{i}(u)$ for $u \in A_{i}, v \in A_{j}$ for $i, j=1, \ldots, N$, and satisfy the estimate $\left\|T_{i}(u)\right\| \leq k_{i}\|x\|$ for $u \in A_{i}, i=1, \ldots, N$. Then we have the decomposition

$$
\mathcal{H}=\mathcal{H}_{1}+\ldots+\mathcal{H}_{2^{N}}
$$


where each $\mathcal{H}_{\alpha}, \alpha=1, \ldots, 2^{N}$, is a closed subspace of $\mathcal{H}$ invariant for $T_{i}$, $i=1, \ldots, N$, and $T_{i}$ restricted to $\mathcal{H}_{\alpha}$ is either $T_{i}^{\mathcal{\mathcal { N } _ { i }}}$ or $T_{i}^{\mathcal{\mathcal { N } _ { i } ^ { \mathrm { s } }}}$. The decomposition (3.17) contains all possible combinations of absolutely continuous and singular parts of representations $T_{i}(i=1, \ldots, N)$ and every such combination appears in (3.17) only once. Moreover, for every $i$ and $\alpha$ we have the estimate

$$
\left\|\left.T_{i}\right|_{\mathcal{H}_{\alpha}}(u)\right\| \leq k_{1} \ldots k_{N}\|u\|, \quad u \in A_{i} .
$$

In the case when $k_{1}=\ldots=k_{N}=1$, the decomposition (3.17) is orthogonal.

4. Commuting contractions. Theorem 3.3 is especially useful to get a Lebesgue-type decomposition of $N$-tuples of commuting contractions in the general situation when we cannot assume any von Neumann inequality.

Any contraction $T$ on a Hilbert space has a unitary dilation, and consequently satisfies the von Neumann inequality $\|p(T)\| \leq\|p\|$ where $p$ is any analytic polynomial and $\|p\|$ denotes its supnorm on the unit disc. Moreover we have the Lebesgue-type decomposition

$$
T=T_{\mathrm{a}} \oplus T_{\mathrm{s}},
$$

where $T_{\mathrm{a}}$ is the orthogonal sum of a completely nonunitary contraction and a unitary operator with spectral measure absolutely continuous with respect to the Lebesgue measure on the unit circle, while $T_{\mathrm{s}}$ is unitary with spectral measure singular with respect to the Lebesgue measure on the unit circle.

For some $N \in \mathbb{N}$, let $T=\left(T_{1}, \ldots, T_{N}\right)$ be an $N$-tuple of commuting contractions on $\mathcal{H}$. Lebesgue-type decompositions for $T$ are known in the case when $T$ satisfies the von Neumann inequality (see [6]).

Theorem 3.3 allows us to get results of that type also in the general case. Every single contraction $T_{j}$ satisfies the von Neumann inequality, and consequently it generates a contractive representation of the disc algebra $A(\mathbb{D})$ in $L(\mathcal{H})$. Since the contractions $T_{j}$ commute, the representations they generate also commute, and applying Theorem 3.3 , we get

TheOREM 4.1. Let $T=\left(T_{1}, \ldots, T_{N}\right)$ be an $N$-tuple of commuting contractions on $\mathcal{H}$, i.e. $T_{i} T_{j}=T_{j} T_{i}$ for $i, j=1, \ldots, N$. Then we have the orthogonal decomposition

$$
\mathcal{H}=\bigoplus_{\alpha=1}^{2^{N}} \mathcal{H}_{\alpha},
$$

where each $\mathcal{H}_{\alpha}$ is a closed subspace of $\mathcal{H}$ reducing $T_{j}, j=1, \ldots, N$, and $T_{j}$ restricted to $\mathcal{H}_{\alpha}$ is either $\left(T_{j}\right)_{\mathrm{a}}$ or $\left(T_{j}\right)_{\mathrm{s}}$. The decomposition (4.20) contains all possible combinations of absolutely continuous and singular parts of the operators $T_{j}(j=1, \ldots, N)$ and every such combination appears in (4.20) only once. 
5. Absolute continuity and singularity. Recall that a contraction $T$ on a Hilbert space is said to be absolutely continuous if it is equal to its absolutely continuous part in the Lebesgue decomposition (4.19). The representation generated by such a contraction has a collection of elementary measures absolutely continuous with respect to Lebesgue measure on the unit circle.

In the case of $N$-tuples $T=\left(T_{1}, \ldots, T_{N}\right)$ of commuting contractions we can talk about their absolute continuity only if they satisfy the von Neumann inequality $\|p(T)\| \leq k\|p\|$ where $p$ is any analytic polynomial of $N$ variables and $k$ is a positive constant. In such a case the $N$-tuple generates a representation of the polydisc algebra $A\left(\mathbb{D}^{N}\right)$ in $L(\mathcal{H})$. If the representation has a collection of elementary measures absolutely continuous with respect to the band of measures generated by all the representing measures for points in the open polydisc then we say that the $N$-tuple is absolutely continuous (see $[9$, Sec. 4]).

In the general case, i.e. without any von Neumann inequality, the above definition cannot be applied. Now we define another property which can always be considered. We say that a given $N$-tuple $T=\left(T_{1}, \ldots, T_{N}\right)$ of commuting contractions is singular if $T_{1}, \ldots, T_{N}$ are unitary with a common spectral measure $E$ on $(\partial \mathbb{D})^{N}$ singular to all measures annihilating $A\left(\mathbb{D}^{N}\right)$. (Recall that $E$ is said to be a common spectral measure of $T_{1}, \ldots, T_{N}$ if $p(T)=\int p d E$ for any polynomial of $N$ variables.) We say that $\mathcal{H}^{\prime} \subset \mathcal{H}$ is a subsingular subspace for $T$ if $\mathcal{H}^{\prime}$ reduces all $T_{1}, \ldots, T_{N}$ and there is a subtuple $T_{j_{1}}, \ldots, T_{j_{k}}, 1 \leq j_{1}<\ldots<j_{k} \leq N$, whose restriction to $\mathcal{H}^{\prime}$ is singular (with respect to the polydisc $\mathbb{D}^{k}$ ). The $N$-tuple $T=\left(T_{1}, \ldots, T_{N}\right)$ is said to be totally nonsingular (abbr. t.n.s.) if it has no nonzero subsingular subspace.

In the case when $T$ satisfies the von Neumann inequality with constant one, i.e. $\|p(T)\| \leq\|p\|$ for all analytic polynomials $p$ of $N$ variables, the property of being totally nonsingular is, by Theorem 1 of [6], equivalent to the absolute continuity (total nonsingularity is called in [6] property $F$ ). On the other hand, the absolute continuity is, by the Main Theorem of [7], equivalent to the following Apostol condition:

$$
\lim _{n \rightarrow \infty} \sup \left|\left\langle p\left(T_{1}, \ldots, T_{N}\right) T_{j}^{n} x, y\right\rangle\right|=0 \quad \text { for } x, y \in \mathcal{H}, j=1, \ldots, N,
$$

where the supremum is taken over all complex analytic polynomials $p$ of $N$ variables with $\|p\| \leq 1$.

In the general case (i.e. without the von Neumann inequality) we can show the following

TheOREM 5.1. Any N-tuple of commuting contractions which satisfies the Apostol condition (5.21) is totally nonsingular. 
Proof. The proof is just a part of the proof of the Main Theorem of [7], but we give it here for the sake of completeness.

We argue by contradiction. Assume that, on the contrary, there is a subtuple $T^{\prime}=\left(T_{j_{1}}, \ldots, T_{j_{m}}\right)$ of $T(1 \leq m \leq N)$ with a closed subspace $\mathcal{H}^{\prime}$ of $\mathcal{H}$ reducing $T^{\prime}$ to unitary operators with a common spectral measure $E$ on $(\partial \mathbb{D})^{m}$ singular to the set of all measures annihilating $A\left(\mathbb{D}^{m}\right)$ (we denote this set by $\left.A\left(\mathbb{D}^{m}\right)^{\perp}\right)$. We should show that $\mathcal{H}^{\prime}=\{0\}$.

Assume $\mathfrak{H}^{\prime} \neq\{0\}$. To simplify the notation, we write $1, \ldots, m$ instead of $j_{1}, \ldots, j_{m}$. Consider

$$
\left\langle f\left(T^{\prime}\right) T_{m}^{n} x, x\right\rangle=\int f\left(z_{1}, \ldots, z_{m}\right) z_{m}^{n} d\left\langle E\left(z_{1}, \ldots, z_{m}\right) x, x\right\rangle,
$$

for any $f \in A\left(\mathbb{D}^{m}\right)$ and $x \in \mathcal{H}^{\prime}$. Take $\varepsilon>0$ and $n \in \mathbb{N}$. Denote by $\mathcal{S}$ the band of all measures singular to $A\left(\mathbb{D}^{m}\right)^{\perp}$. By the definition of $\mathcal{S}, A\left(\mathbb{D}^{m}\right)$ is weakstar dense in the dual $\mathcal{S}^{*}$ of $\mathcal{S}$, since no measure in $\mathcal{S}$ annihilates $A\left(\mathbb{D}^{m}\right)$. Moreover, since the band $\mathcal{S}$ is reducing, the unit ball of $A\left(\mathbb{D}^{m}\right)$ is weak-star dense in the unit ball of $\mathcal{S}^{*}$ (see Lemma 2.2 of [7]). Consequently, since the function $z_{m}^{-n}$ can be considered as an element of $\mathcal{S}^{*}$, and the measure $z_{m}^{n}\langle E(\cdot) x, x\rangle$ is in $\mathcal{S}$, we can find a polynomial $q$ of norm 1 such that

$$
\left|\int q(z) z_{m}^{n} d\langle E(z) x, x\rangle-\int z_{m}^{-n} z_{m}^{n} d\langle E(z) x, x\rangle\right|<\varepsilon .
$$

Thus, since

$$
\int_{(\partial \mathbb{D})^{m}} z_{m}^{-n} z_{m}^{n} d\langle E(z) x, x\rangle=1
$$

we have

$$
\sup _{\|p\| \leq 1}\left|\left\langle p(T) T_{m}^{n} x, x\right\rangle\right| \geq\left|\left\langle q\left(T^{\prime}\right) T_{m}^{n} x, x\right\rangle\right|=\left|\int q(z) z_{m}^{n} d\langle E(z) x, x\rangle\right|>1-\varepsilon .
$$

Since $n$ was arbitrary, the condition (5.21) is not satisfied, which finishes the proof.

A subspace $\mathcal{K} \subset \mathcal{H}$ is said to be hyperreducing for a collection of bounded linear operators on $\mathcal{H}$ if it is reducing for its commutant. We say that a given $N$-tuple of commuting contractions is proper if none of its components is multiplication by a constant. From Theorem 4.1 we can deduce the following result:

THEOREM 5.2. If a proper $N$-tuple of commuting contractions on $\mathcal{H}$ has a nonzero subsingular subspace then it also has a nontrivial hyperreducing subspace.

Proof. Let $T$ be a given proper $N$-tuple, $\mathcal{K}$ its subsingular subspace and $T^{\prime}$ a subtuple of $T$ which is singular on $\mathcal{K}$. The subtuple $T^{\prime}$ has a common spectral measure $F$ on some $m$-dimensional torus $(\partial \mathbb{D})^{m}$ such that $F$ is singular to $A\left(\mathbb{D}^{m}\right)^{\perp}$. Since $T^{\prime}$ is proper, $F$ is not concentrated at one point. 
Consequently, by the regularity of $F$, there are two disjoint compact subsets $E_{1}, E_{2}$ of $(\partial \mathbb{D})^{m}$ on which $F$ is nonzero. By the singularity of $F$, the sets $E_{1}, E_{2}$ can be chosen so that they are peak interpolation sets for $A\left(\mathbb{D}^{m}\right)$. Hence for $i=1,2$, the band of measures which are supported on $E_{i}$ is reducing for $A\left(\mathbb{D}^{m}\right)$. So, by Theorem 2.2 , there exists a nontrivial closed subspace of $\mathcal{H}$ which is reducing for the commutant of $T^{\prime}$ and consequently also for the commutant of $T$.

Note that if the subspace $\mathcal{K}$ in the proof above is proper and it is the maximal subspace on which the subtuple $T^{\prime}$ is singular then, by Theorem 2.2, it is also hyperreducing. From Theorem 5.2 we can deduce the following corollary for $N$-tuples without nontrivial invariant subspaces, if such an object exists:

Corollary 5.3. Any proper $N$-tuple of commuting contractions without common nontrivial invariant subspaces is totally nonsingular.

\section{References}

[1] T. Ando, On a pair of commutative contractions, Acta Sci. Math. (Szeged) 24 (1963), $88-90$.

[2] O. B. Bekken, Rational approximation on product sets, Trans. Amer. Math. Soc. 191 (1974), 301-316.

[3] B. J. Cole and T. W. Gamelin, Tight uniform algebras of analytic functions, J. Funct. Anal. 46 (1982), 158-220.

[4] J. B. Conway, The Theory of Subnormal Operators, Amer. Math. Soc., Providence, RI, 1991.

[5] T. W. Gamelin, Uniform Algebras, Prentice-Hall, Englewood Cliffs, NJ, 1969.

[6] M. Kosiek, Representation generated by a finite number of Hilbert space operators, Ann. Polon. Math. 44 (1984), 309-315.

[7] M. Kosiek and A. Octavio, Representations of $H^{\infty}\left(\mathbb{D}^{N}\right)$ and absolute continuity for $N$-tuples of contractions, Houston J. Math. 30 (1997), 529-537.

[8] M. Kosiek, A. Octavio and M. Ptak, On the reflexivity of pairs of contractions, Proc. Amer. Math. Soc. 123 (1995), 1229-1236.

[9] M. Kosiek and M. Ptak, Reflexivity of $N$-tuples of contractions with rich joint left essential spectrum, Integral Equations Oper. Theory 13 (1990), 395-420.

[10] M. Kosiek and M. Zajac, Lebesgue-type decompositions of pairs of commuting contractions, Math. Balkanica 4 (1990), 16-21.

[11] W. Mlak, Decompositions and extensions of operator valued representations of function algebras, Acta Sci. Math. (Szeged) 30 (1969), 181-193.

[12] - Intertwining operators, Studia Math. 43 (1972), 219-233.

[13] V. Müller and F. H. Vasilescu, Standard models for some commuting multioperators, Proc. Amer. Math. Soc. 117 (1993), 979-989.

[14] W. Rudin, Function Theory in the Unit Ball of $\mathbb{C}^{n}$, Springer, New York, 1980.

[15] K. Rudol, Separation property and uniqueness of some spectra on $H^{\infty}$, Bull. Polish Acad. Sci. Math. 41 (1993), 51-54. 
[16] F. H. Szafraniec, Decompositions of non-contractive operator-valued representations of Banach algebras, Studia Math. 42 (1972), 97-108.

Institute of Mathematics

Jagiellonian University

Reymonta 4

30-059 Kraków, Poland

E-mail: mko@im.uj.edu.pl 